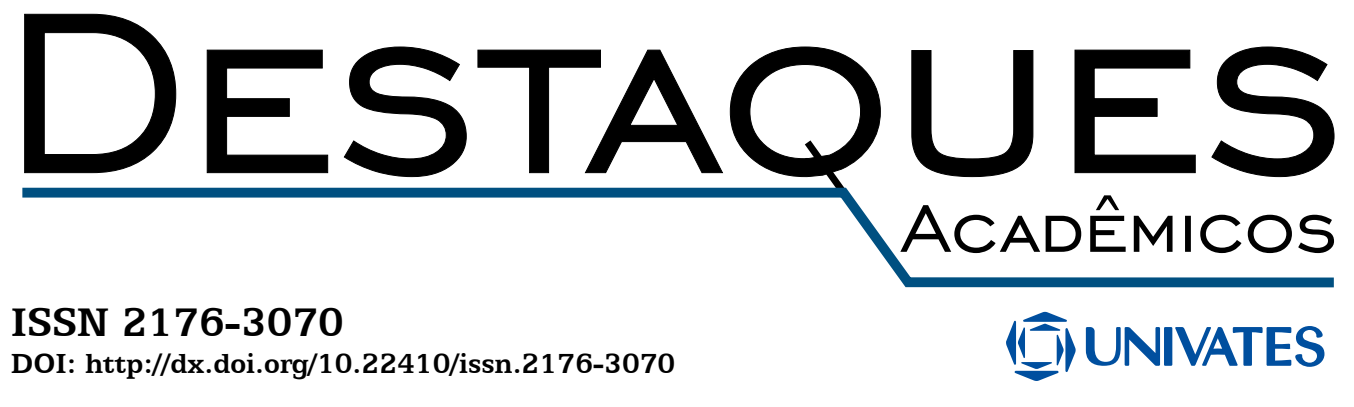

\title{
CIÊNCIAS EXATAS E TECNOLÓGICAS
}

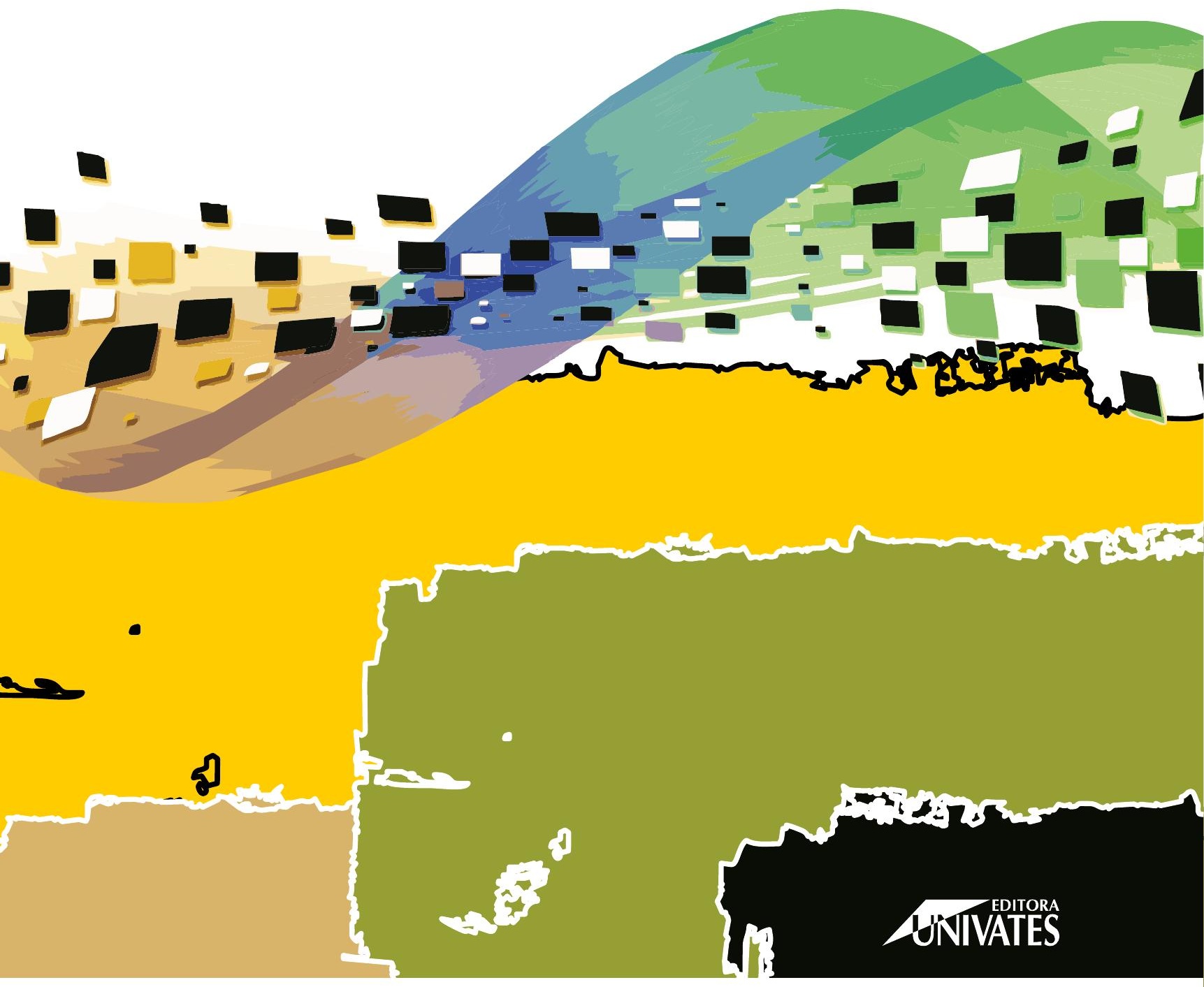




\section{VIIIIUNIVATES}

Universidade do Vale do Taquari - Univates

Reitor: Prof. Me. Ney José Lazzari

Vice-Reitor e Presidente da Fuvates: Prof. Dr. Carlos Cândido da Silva Cyrne

Pró-Reitora de Pesquisa, Extensão e Pós-Graduação: Profa. Dra. Maria Madalena Dullius

Pró-Reitora de Ensino: Profa. Dra. Fernanda Storck Pinheiro

Pró-Reitora de Desenvolvimento Institucional: Profa. Dra. Júlia Elisabete Barden

Pró-Reitor Administrativo: Prof. Me. Oto Roberto Moerschbaecher

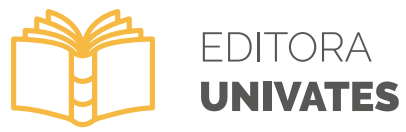

\section{Editora Univates}

Coordenação: Ana Paula Lisboa Monteiro

Editoração e capa: Glauber Röhrig e Marlon Alceu Cristófoli

\section{Conselho Editorial da Editora Univates}

\section{Titulares}

Alexandre André Feil

André Anjos da Silva

Fernanda Rocha da Trindade

João Miguel Back

Sônia Elisa Marchi Gonzatti

\author{
Suplentes \\ Fernanda Cristina Wiebusch Sindelar \\ Claudete Rempel \\ Adriane Pozzobon \\ Rogério José Schuck \\ Evandro Franzen
}

Avelino Talini, 171 - Bairro Universitário - Lajeado - RS, Brasil

Fone: (51) 3714-7024 / Fone: (51) 3714-7000, R.: 5984

editora@univates.br / http://www.univates.br/editora

D476 Destaques Acadêmicos / Centro Universitário Univates - Vol. 1, no. 1 (2009) - Lajeado, RS: Ed. da Univates, 2020.

v. 12 , n. 4, 2020.

Instituição mudou o nome para Universidade do Vale do Taquari - Univates em 2017.

Trimestral

ISSN 2176-3070

1. Ensino. 2. Ensino multidisciplinar. I. Universidade do Vale do Taquari - Univates.

CDU: 378

Catalogação na publicação - Biblioteca da Univates Bibliotecária Andrieli Mara Lanferdini - CRB 10/2279

Os artigos são de exclusiva responsabilidade do(s) autor(es).

(C) Fundação Vale do Taquari de Educação e Desenvolvimento Social - FUVATES 


\section{DESTAOUES}

Conselho Editorial da Revista

Cristiane Antonia Hauschild

João Miguel Back

Magali Teresinha Quevedo Grave

Marlon Dalmoro

\section{Coordenadora desta edição}

Cristiane Antonia Hauschild

Pareceristas desta edição

Alexandre Stürmer Wolf

Carlos Candido da Silva Cyrne

Cristiane Antonia Hauschild

Daniel Neutzling Lehn

Debora Pedroso Righi

Edson Moacir Ahlert

Evandro Franzen

Jane Herber

Liciane Diehl

Mailson Scherer

Marcelo Arioli Heck

Marcelo Arioli Heck

Márcia Jussara Hepp Rehfeldt

Maria Cristina de Almeida Silva

Marli Teresinha Quartieri

Rafael Mascolo

Rebeca Jéssica Schmitz

Tiago Weizenmann 


\section{APRESENTAÇÃO}

Prezado(a) leitor(a), 2020 foi um ano atípico para o mundo, com uma profunda tristeza para uma boa parcela da população, que perdeu entes queridos em razão da pandemia da Covid 19 causada pelo novo coronavírus, com quem nos solidarizamos. Mas também mostrou a importância da qualificação e do desenvolvimento da Ciência e da Tecnologia.

Por aqui, nos adaptamos rapidamente para continuar estudos e pesquisas com segurança. Nesse sentido, a Revista Destaques Acadêmicos da Universidade do Vale do Taquari - Univates, em sua quarta edição de 2020, apresenta 19 artigos ligados à área de Ciências Exatas e Tecnológicas, de forma a contribuir com a disseminação da Ciência e da Tecnologia. Os artigos abordam uma variedade de temáticas que podem ser conferidas na Figura 1, escritos por estudantes, professores e pesquisadores.

Figura 1 - Nuvem de palavras com as palavras-chaves dos artigos selecionados ${ }^{1}$

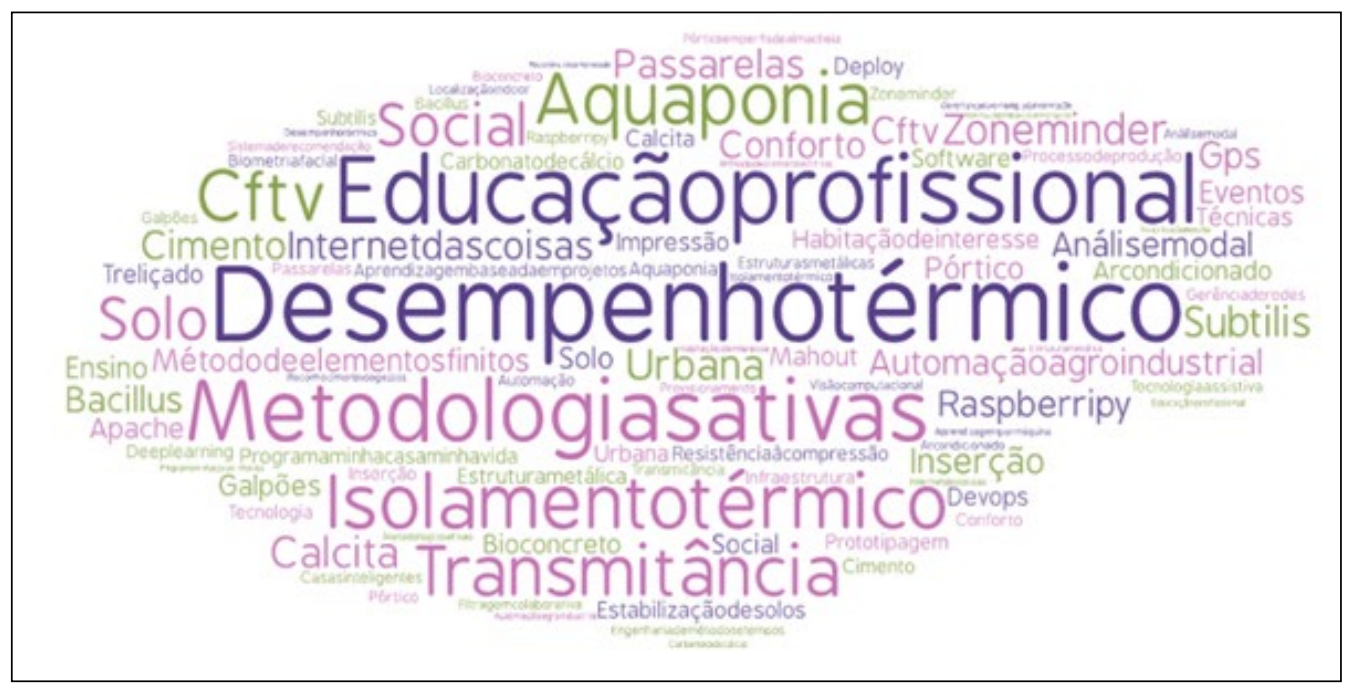

Fonte: da autora.

Gratidão ao coletivo de pareceristas.

Desejamos uma ótima leitura!

Profa. Dra. Cristiane Antonia Hauschild

Coordenadora desta edição

1 Os espaços foram omitidos quando as expressões são compostas por mais de uma palavra. 


\section{SUMÁRIO}

DESEMPENHO TÉRMICO E ACÚSTICO: AVALIAÇÃO DAS

PROPOSTAS DE MELHORIAS DE DESEMPENHO TÉRMICO DE UMA

EDIFICAÇÃO UNIFAMILIAR MINHA CASA MINHA VIDA ……..................7

Adriano Inéia, Angélica Ghiggi, Rodrigo Spinelli

SISTEMA DE MONITORAMENTO AUTOMATIZADO PARA

CONTROLE DE QUALIDADE DE ÁGUA EM SISTEMA AQUAPÔNICO ....32

Alessandro Antoniolli, Edson Moacir Ahlert

IMPLEMENTAÇÃO DE SISTEMA DE VIDEOMONITORAMENTO DE

BAIXO CUSTO UTILIZANDO ZONEMINDER E CÂMERAS IP.

Robinson Stürmer, Edson Moacir Ahlert

ANÁLISE MODAL NUMÉRICA DE UMA PASSARELA DE PEDESTRES.....69

Guilherme Leite Lago, Gabriel Birck, Marcelo André Toso

UM OLHAR SOBRE A RECENTE PRODUÇÃO HABITACIONAL DE

INTERESSE SOCIAL EM LAJEADO-RS

Tainá Manfredini, Alice Rauber Gonçalves

APRENDIZAGEM BASEADA EM PROJETOS COMO METODOLOGIA

DE ENSINO NO CURSO TÉCNICO EM EDIFICAÇÕES 104

Francine Maria Sauter Gutjahr, Maria Claudete Schorr

INFLUÊNCIA DO TEOR DE CIMENTO NA RESISTÊNCIA À COMPRESSÃO SIMPLES DE UM SOLO RESIDUAL CIMENTADO

ARTIFICIALMENTE

Thaise Canton Somensi, Guilherme Togni, Augusto Armani, Helena Batista Leon,

João Rodrigo Guerreiro Mattos, Emanuele Amanda Gauer

ANÁLISE DA INFLUÊNCIA DE MATERIAIS E MÉTODOS

CONSTRUTIVOS NO DESEMPENHO TÉRMICO EM HABITAÇÃO

SOCIAL NO MUNICÍPIO DE LAJEADO/RS

Alfredo Henrique Giovanaz, Isabel Kristiner, João Marcos Sartori

UTILIZAÇÃO DE MICRORGANISMO AUTÓGENO PARA A RECUPERAÇÃO DE FISSURAS EM CORPOS DE PROVA DE ARGAMASSA 143

Graciela Mânica, Claucia Fernanda Volken de Souza 
COMPARATIVO DE PESO DOS PÓRTICOS DE UM GALPÃO

CONSIDERANDO SISTEMA EM TRELIÇASE EM PERFIS DE ALMA

CHEIA 163

Alysson Bianchini, Rebeca Jéssica Schmitz

IMPRESSÃO 3D APLICADA À TECNOLOGIA ASSISTIVA .181

Eloar Froboese da Silva, Lucas Menin da Silva, Vinícius Gonçalves Deon,

Marcelo André Toso

ENGENHARIA DE MÉTODOS E TEMPOS APLICADA NO PROCESSO

DE PRODUÇÃO DE ESTRUTURAS METÁLICAS.

André Mendonça da Costa

SISTEMA DE RECOMENDAÇÃO DE EVENTOS UTILIZANDO O

FRAMEWORK APACHE MAHOUT .216

Willian Valmorbida, Christian Rodolpho Bugs

CONTROLE DE DISPOSITIVOS EM CASAS INTELIGENTES POR MEIO

DO RECONHECIMENTO DE GESTOS E BIOMETRIA FACIAL COM

USO DE DEEP LEARNING. 235

Willian Valmorbida, Klaus Fernando Etgeton

ESTUDO DE PROPOSTAS PARA UM SISTEMA DE TRAÇÃO 4X4 EM UM VEÍCULO DO TIPO BAJA SAE .253

Eduardo Zotti, Misael David Hattge Lenz, Ricson Rocha de Souza

BLUEPATH: SISTEMA DE LOCALIZAÇÃO INDOOR .266

Raul Silvério Mittelstadt, Fabrício Pretto

AUTOM8 - FERRAMENTA WEB PARA GERENCIAMENTO, MANUTENÇÃO E ATUALIZAÇÃO REMOTA DE SISTEMAS OPERACIONAIS E APLICATIVOS. 277

Heryk Gasparini, Fabrício Pretto

IMPLEMENTAÇÃO DE INFRAESTRUTURA COMO CÓDIGO PARA PROVISIONAMENTO E DEPLOY DE APLICAÇÕES .296

Jones Luis Noll, Fabrício Pretto

A UTILIZAÇÃO DE METODOLOGIAS ATIVAS NOS CURSOS DO CENTRO DE EDUCAÇÃO PROFISSIONAL DA UNIVERSIDADE DO VALE DO TAQUARI - UNIVATES 316

Daniela Luisa Scheibel, Maria Claudete Schorr 